\title{
Multi Criteria Decision Making in Fuzzy Description Logics: A First Step
}

\author{
Umberto Straccia \\ Istituto di Scienza e Tecnologie dell'Informazione (ISTI - CNR), Pisa, Italy \\ straccia@isti.cnr.it
}

\begin{abstract}
Fuzzy Description Logics are logics which allow to deal with structured knowledge affected by vagueness. Although a relatively important amount of work has been carried out in the last years, fuzzy DLs are open to be extended with several features worked out in other fields. In this work, we start addressing the problem of incorporating Multi-Criteria Decision Making (MCDM) into fuzzy Description Logics and, thus, start an investigation about offering the possibility of a fuzzy ontology assisted approach to decision making.
\end{abstract}

\section{Introduction}

Description Logics (DLs) [1] play a key role in the design of Ontologies. An ontology consists of a hierarchical description of important concepts in a particular domain, along with the description of the properties (of the instances) of each concept. DLs play a particular role in this context as they are essentially the theoretical counterpart of the Web Ontology Language OWL DL, a state of the art language to specify ontologies.

It is well-known that "classical" ontology languages are not appropriate to deal with fuzzy knowledge, which is inherent to several real world domains [1012. Fuzzy ontologies emerge as useful in several applications, such as (multimedia) information retrieval, image interpretation, ontology mapping, matchmaking and the Semantic Web [8]. So far, several fuzzy extensions of DLs can be found in the literature (see the survey in [8]) and some fuzzy DL reasoners have been implemented, such as FUZZYDL [3], DeLorean [2] or Fire 9].

In this work, we start investigating about using fuzzy DLs as a fuzzy ontology support for Multi-Criteria Decision Making (MCDM) [13], which is among one of the most well known branches of decision making. Roughly, MCDM is the study of identifying and choosing alternatives based on the values and preferences of the decision maker. Making a decision implies that there are alternative choices to be considered and to choose the one that best fits with our goals, objectives, desires, values, and so on. Our work should be understood as an attempt in using fuzzy DLs and, thus, fuzzy ontologies, for knowledge assisted decision making. While there is a large literature on fuzzy MCDM [6] and fuzzy DLs [8, to the best of our knowledge, this is the first time such a combination is addressed.

We proceed as follows. Section 2 (resp. Section [3) will provide the basic concepts related to mathematical fuzzy logic (resp. MCDM) we will rely on, Section 4 
specifies a minimal fuzzy DL to deal with MCDM and illustrates some examples. Section 5 concludes and describes some future work.

\section{Fuzzy Sets and Mathematical Fuzzy Logic Basics}

In Mathematical Fuzzy Logic [5, the convention prescribing that a statement is either true or false is changed and is a matter of degree measured on an ordered scale $\mathcal{S}$ that is no longer $\{0,1\}$, but usually the unit interval $[0,1]$. This degree of fit is called degree of truth of the statement $\phi$ in the interpretation $\mathcal{I}$. In this section, for illustrative purposes, fuzzy statements have the form $\phi \geqslant l$ or $\phi \leqslant u$, where $l, u \in[0,1]$ (see, e.g. [5]) and $\phi$ is a statement. Fuzzy statements encode that the degree of truth of $\phi$ is at least equal to $l$ resp. at most equal to $u$. A fuzzy interpretation $\mathcal{I}$ maps each basic statement $p_{i}$ into $[0,1]$ and is then extended inductively to all statements: $\mathcal{I}(\phi \wedge \psi)=\mathcal{I}(\phi) \otimes \mathcal{I}(\psi), \mathcal{I}(\phi \vee \psi)=\mathcal{I}(\phi) \oplus \mathcal{I}(\psi)$, $\mathcal{I}(\phi \rightarrow \psi)=\mathcal{I}(\phi) \Rightarrow \mathcal{I}(\psi), \mathcal{I}(\neg \phi)=\ominus \mathcal{I}(\phi), \mathcal{I}(\exists x . \phi(x))=\sup _{a \in \Delta^{I}} \mathcal{I}(\phi(a))$, $\mathcal{I}(\forall x . \phi(x))=\inf _{a \in \Delta^{\mathcal{I}}} \mathcal{I}(\phi(a))$, where $\Delta^{\mathcal{I}}$ is the domain of $\mathcal{I}$, and $\otimes, \oplus, \Rightarrow$, and $\ominus$ are $t$-norms, $t$-conorms, implication functions, and negation functions, respectively, which extend the classical Boolean conjunction, disjunction, implication, and negation, respectively, to the fuzzy case. One usually distinguishes three different logics (see below), namely Lukasiewicz, Gödel, and Product logic [5. Zadeh "logic", namely $a \otimes b=\min (a, b), a \oplus b=\max (a, b), \ominus a=1-a$ and $a \Rightarrow b=\max (1-a, b)$ is entailed by Łukasiewicz logic.

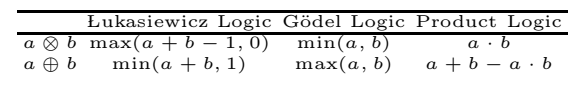

\begin{tabular}{|c|c|c|c|}
\hline & Łukasiewicz Logic & Gödel Logic & Product Logic \\
\hline$a=$ & $\min (1-a+b, 1)$ & $\begin{cases}1 & \text { if } a \leq b \\
b & \text { otherwise }\end{cases}$ & $\min (1$ \\
\hline$\theta$ & $1-$ & $\begin{cases}1 & \text { if } a=0 \\
0 & \text { otherwise }\end{cases}$ & $\begin{cases}1 & \text { if } a=0 \\
0 & \text { otherwise }\end{cases}$ \\
\hline
\end{tabular}

In fuzzy set theory [7, a fuzzy set $R$ over a countable crisp set $X$ is a function $R: X \rightarrow[0,1]$. A (binary) fuzzy relation $R$ over two countable crisp sets $X$ and $Y$ is a function $R: X \times Y \rightarrow[0,1]$. We say that $R$ is functional iff $R$ is a partial function $R: X \times Y \rightarrow\{0,1\}$ such that for each $x \in X$ there is unique $y \in X$ where $R(x, y)$ is defined. The degree of subsumption between two fuzzy sets $A$ and $B$ is defined as $\inf _{x \in X} A(x) \Rightarrow B(x)$ and may be seen as the degree of the FOL formula $\forall x . A(x) \rightarrow B(x)$, while the degree of overlap between two fuzzy sets $A$ and $B$ is defined as $\sup _{x \in X} A(x) \wedge B(x)$ and may be seen as the degree of the FOL formula $\exists x \cdot A(x) \wedge B(x)$.

The notions of satisfiability and logical consequence are defined in the standard way. A fuzzy interpretation $\mathcal{I}$ satisfies a fuzzy statement $\phi \geqslant l$ (resp., $\phi \leqslant u$ ) or $\mathcal{I}$ is a model of $\phi \geqslant l$ (resp., $\phi \leqslant u$ ), denoted $\mathcal{I} \mid \phi \geqslant l$ (resp., $\mathcal{I} \models \phi \leqslant u$ ), iff $\mathcal{I}(\phi) \geqslant l$ (resp., $\mathcal{I}(\phi) \leqslant u)$. Furthermore, $\phi \geqslant l$ is a tight logical consequence of a set of fuzzy statements $\mathcal{K}$ iff $l$ is the infimum of $\mathcal{I}(\phi)$ subject to all models $\mathcal{I}$ of $\mathcal{K}$. The latter value is equivalent to $l=\sup \{r \mid \mathcal{K} \models \phi \geqslant r\}$, it is called Best Entailment Degree $(\mathrm{BED})$, and is denoted bed( $\mathcal{K}, \phi)$, while the Best Satisfiability Degree (BSD), denoted as $\operatorname{bsd}(\mathcal{K}, \phi)$, is defined as $\sup _{\mathcal{I}=\mathcal{K}} \mathcal{I}(\phi)$. 


\section{MCDM Basics}

The area of MCDM is quite vast and we cannot address all the addressed issues here. We will focus on the basic notions that are of importance in MCDM and a simple MCDM method to be used here.

Usually, alternatives represent different choices of action available to the decision maker and is assumed to be finite in our case. The decision criteria represent the different dimensions from which the alternatives can be viewed (a decision criteria is also referred to as goals or attributes). Most of the MCDM methods require the criteria be assigned decision weights of importance. Usually, these weights are normalized to add up to one.

A MCDM problem of $m$ criteria and $n$ alternatives is informally as follows: let $\mathbf{A}=\left\{A_{1}, \ldots, A_{n}\right\}$ be a set of $n$ decision alternatives and let $\mathbf{C}=\left\{C_{1}, \ldots, C_{m}\right\}$ be a set of $m$ criteria according to which the desirability of an action is judged. Determine the optimal alternative $A^{*}$ with the highest degree of desirability.

A standard feature of MCDM methods is that a MCDM problem can be expressed by means of a decision matrix, as shown below.

\begin{tabular}{|c|c|c|c|c|c|c|}
\hline & & \multicolumn{5}{|c|}{ Criteria } \\
\hline & & $w_{1}$ & $w_{2}$ & $\cdot$ & $\cdot$ & $w_{m}$ \\
\hline \multicolumn{2}{|c|}{ Alternatives } & $C_{1}$ & $C_{2}$ & $\cdot$ & $\cdot$ & $C_{m}$ \\
\hline \hline$x_{1}$ & $A_{1}$ & $a_{11}$ & $a_{12}$ & $\cdot$ & $\cdot$ & $a_{1 m}$ \\
$x_{2}$ & $A_{2}$ & $a_{21}$ & $a_{22}$ & $\cdot$ & $\cdot$ & $a_{2 m}$ \\
$\cdot$ & $\cdot$ & $\cdot$ & $\cdot$ & $\cdot$ & $\cdot$ & $\cdot$ \\
$\cdot$ & $\cdot$ & $\cdot$ & $\cdot$ & $\cdot$ & $\cdot$ & $\cdot$ \\
$x_{n}$ & $A_{n}$ & $a_{n 1}$ & $a_{n 2}$ & $\cdot$ & $\cdot$ & $a_{n m}$ \\
\hline
\end{tabular}

In the matrix each column belongs to a criterion $C_{j}$ and each row describes the performance of an alternative $A_{i}$. The score $a_{i j}$ describes the performance of alternative $A_{i}$ against criterion $C_{j}$. The weights $w_{1}, \ldots, w_{m}$ are assigned to the criteria. Weight $w_{j}$ reflects the relative importance of criteria $C_{j}$ to the decision, and is assumed to be positive and normalized, i.e. $1=\sum_{j=1}^{m} w_{j}$. The weights of the criteria are usually determined on subjective basis and may also be seen as a kind of profit of the criteria. They represent the opinion of a single decision maker or synthesize the opinions of a group of experts. Not surprisingly, there is a large literature on methods to assign weights (see, e.g. 13]). For illustrative purpose, we illustrate here a method based on pairwise comparison of the criteria to determine the weights. It consists in comparing elements (criteria) $X_{i}$ with $X_{j}$ $(1 \leq i, j \leq k)$ and judge how much they contribute to the overall objective. The judgment consists in assigning a number $w_{i j} \in[1,9]$, called Intensity of Importance, selected according the following table

\begin{tabular}{|c|c|l|}
\hline Intensity & Definition & Explanation \\
\hline 1 & Equal Importance & $\begin{array}{l}\text { Two elements contribute equally to the objec- } \\
\text { tive }\end{array}$ \\
\hline 3 & Moderate Importance & $\begin{array}{l}\text { Experience and judgement slightly favor one } \\
\text { element over the other }\end{array}$ \\
\hline 5 & Strong Importance & $\begin{array}{l}\text { Experience and judgement strongly favor one } \\
\text { element over the other }\end{array}$ \\
\hline 7 & Very Strong Importance & $\begin{array}{l}\text { One element is favored very strongly over an- } \\
\text { other, its dominance is demonstrated in practice }\end{array}$ \\
\hline 9 & Extreme Importance & $\begin{array}{l}\text { The evidence favoring one element over another } \\
\text { is of the highest possible order of information }\end{array}$ \\
\hline
\end{tabular}


Intermediate values can be used. The weight $w_{i}$ of element $X_{i}$ may be obtained as

$$
\bar{w}_{i}=\left(\prod_{j=1}^{k} w_{i j}\right)^{1 / k}, \bar{w}=\sum_{i=1}^{k} \bar{w}_{i}, \quad \text { and then } w_{i}=\bar{w}_{i} / \bar{w} .
$$

On more on alternatives to determine the data of a decision matrix and their impact, see e.g., 13 .

The values $x_{1}, \ldots, x_{n}$ associated with the alternatives in the decision matrix will be used to denote the final ranking values of the alternatives. Usually, higher ranking value means a better performance of the alternative, so the alternative with the highest ranking value is the best of the alternatives. MCDM techniques can partially or completely rank the alternatives: a single most preferred alternative can be identified or a short list of a limited number of alternatives can be selected for subsequent detailed appraisal. Again, there are many alternative methods to compute the final ranking values from the decision matrix. For illustrative purposes, we present the so-called Weighted Sum Method (WSM), which is among the simplest methods in MCDM, but has the advantage to be easy embedded within fuzzy DLs. Formally, let

$$
x_{i}=\sum_{j=1}^{m} a_{i j} w_{j} \text { for } i=1,2, \ldots, m .
$$

where $x_{i}$ is the the final ranking value of alternative $A_{i}$. The ranking of the alternatives is obtained by ordering the alternatives in descending order with respect to the final ranking value and the optimal alternative $A^{*}$ is the one that maximizes the final ranking value, i.e.

$$
A^{*}=\arg \max _{A_{i}} x_{i} .
$$

We conclude this section by pointing out that in fuzzy MCDM, a principal difference to classical MCDM is due to the fact that weights $w_{i}$ and performance factors $a_{i j}$ are so-called fuzzy numbers [7. A fuzzy number $\tilde{n}$ is a fuzzy set over relas with triangular membership function $\operatorname{tri}(a, b, c)$ and is intended being an approximation of the number $b$. Any real value $n$ is seen as the fuzzy number $\operatorname{tri}(n, n, n)$. The arithmetic operators,,$+- \cdot$ and $\div$ are extended to fuzzy numbers by applying them to the arguments, i.e. for fuzzy numbers $\tilde{n}_{1}=\operatorname{tri}\left(a_{1}, b_{1}, c_{1}\right)$ and $\tilde{n_{2}}=\operatorname{tri}\left(a_{2}, b_{2}, c_{2}\right)$, for operator $* \in\{+, \cdot\}, \tilde{n}_{1} * \tilde{n}_{2}=\operatorname{tri}\left(a_{1} * a_{2}, b_{1} * b_{2}, c_{1} * c_{2}\right)$, while for $* \in\{-, \div\}, \tilde{n}_{1} * \tilde{n}_{2}=\operatorname{tri}\left(a_{1} * c_{2}, b_{1} * b_{2}, c_{1} * a_{2}\right)$. The final rank value is computed as in Eq. 2,

$$
\tilde{x}_{i}=\sum_{j=1}^{m} \tilde{a}_{i j} \cdot \tilde{w}_{j} \text { for } i=1,2, \ldots, m .
$$

As now $\tilde{x}_{i}$ is a fuzzy number, one may apply some defuzzyfication method to it and compere fuzzy numbers based on these values, or one may use some fuzzy number comparison operator to determine the optimal solution (see, e.g. [13]). 


\section{Towards MCDM within Fuzzy Description Logics}

The aim of this section is to show that current fuzzy DLs can be used to deal with (fuzzy) knowledge assisted MCDM (though, the MCDM method needs to be simple so far). For illustrative purposes and for reasons of space, we will just consider a minimal fuzzy DL to deal with the WSM in MCDM.

Syntax. We will present $\mathcal{A L C F}(D)$, which is the basic DL $\mathcal{A L C}$ extended with functional roles (letter $\mathcal{F}$ ) and concrete domains [11] (letter $D$ ).

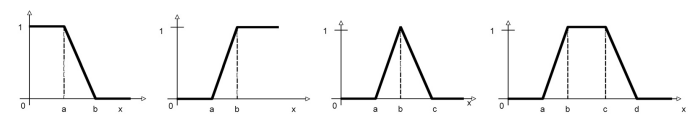

In general, a fuzzy concrete domain (or simply fuzzy domain) [1] is a pair $\left\langle\Delta_{D}, \Phi_{D}\right\rangle$, where $\Delta_{D}$ is an interpretation domain and $\Phi_{D}$ is the set of fuzzy domain predicates $d$ with a predefined arity $n$ and an interpretation $d^{D}: \Delta_{D}^{n} \rightarrow$ $[0,1]$, which is a $n$-ary fuzzy relation over $\Delta_{D}$. In our specific spatial fuzzy DL, we assume that predicates are unary and $\Delta_{D}$ are non-negative real numbers.

Now, consider pairwise disjoint alphabets of concepts names (denoted $A$ ), abstract roles names (denoted $R$ ) and concrete roles names (denoted $T$ ). Within the alphabet of abstract and concrete roles, we have distinguished subsets of abstract functional roles names (denoted $f$ ) and concrete functional roles names (denoted $t$ ), respectively. We call functional roles also features. From a First-Order Logic point of view, concepts may be seen as a formulae with one free variable (and, thus, may be seen as class descriptors), while roles as binary predicates (and, thus, may be used to describe properties of a class). Concepts (denoted $C$ or $D$ ) of the language can be built inductively from atomic concepts $(A)$, top concept $T$, bottom concept $\perp$, abstract roles $(R)$, concrete roles $(T)$ as follows. The syntax of fuzzy concepts (denoted $C, D$ ) is as follows:

$$
C, D:=\top|\perp| A|C \sqcap D| C \sqcup D|\neg C| \forall R . C \mid \exists R . C
$$

Now, the fuzzy DL is extended as follows $[3$ :

$$
\begin{aligned}
C, D & :=\forall T . d|\exists T . d| w_{1} C_{1}+\cdots+w_{k} C_{k} \\
d & :=l s(a, b)|r s(a, b)| \operatorname{tri}(a, b, c) \mid \operatorname{trap}(a, b, c, d)
\end{aligned}
$$

where $v a l$ is an integer, a real or a string depending on the range of the concrete feature $t, w_{i} \in[0,1]_{D}, \sum_{i=1}^{k} w_{i} \leq 1$ and $C_{i}$ are concept:1. E.g., the expression Human $\sqcap(\leq$ has Age 18) will denote the set of humans, which have an age less or equal than 18 , while Human $\sqcap \exists$ hasAge.ls $(10,30)$ will denote the set of young humans (their age is $l s(10,30)$ ).

A Fuzzy Knowledge Base (or fuzzy Ontology) consists of a finite set of fuzzy General Concept Inclusions (fuzzy GCIs), which are expressions of the form $\langle C \sqsubseteq D, n\rangle$ (with informal meaning, the degree of subsumption between concept $C$ and $D$ is not less than $n$ ). In FOL, $\langle C \sqsubseteq D, n\rangle$ may be seen as a fuzzy statement of the form $(\forall x . C(x) \rightarrow D(x)) \geqslant n$ and amounts of asserting that the

\footnotetext{
${ }^{1}$ In 3] we assume $\sum_{i=1}^{k} w_{i}=1$ instead, however, this modification is harmless.
} 
degree of subsumption among $C$ and $D$ is at least $n$. We will use $C=D$ as a shorthand for $\langle C \sqsubseteq D, 1\rangle$ and $\langle D \sqsubseteq C, 1\rangle$.

Semantics. From a semantics point of view, a fuzzy interpretation $\mathcal{I}=\left(\Delta^{\mathcal{I}},{ }^{\mathcal{I}}\right)$ relative to the fuzzy concrete domain $\left\langle\Delta_{D}, \Phi_{D}\right\rangle$, consists of a nonempty set $\Delta^{\mathcal{I}}$ (the domain), disjoint from $\Delta_{D}$, and of a fuzzy interpretation function. I that coincides with $\cdot D$ on every fuzzy concrete predicate, and it assigns: $(i)$ to each abstract concept $C$ a function $C^{\mathcal{I}}: \Delta^{\mathcal{I}} \rightarrow[0,1] ;(i i)$ to each abstract role $R$ a function $R^{\mathcal{I}}: \Delta^{\mathcal{I}} \times \Delta^{\mathcal{I}} \rightarrow[0,1]$; (iii) to each abstract feature $r$ a partial function $r^{\mathcal{I}}: \Delta^{\mathcal{I}} \times \Delta^{\mathcal{I}} \rightarrow\{0,1\}$ such that for all $u \in \Delta^{\mathcal{I}}$ there is an unique $w \in \Delta^{\mathcal{I}}$ on which $r^{\mathcal{I}}(u, w)$ is defined; (iv) to each concrete role $T$ a function $T^{\mathcal{I}}: \Delta^{\mathcal{I}} \times \Delta_{D} \rightarrow[0,1]$; $(v)$ to each concrete feature $t$ a partial function $t^{\mathcal{I}}: \Delta^{\mathcal{I}} \times \Delta_{D} \rightarrow\{0,1\}$ such that for all $u \in \Delta^{\mathcal{I}}$ there is an unique $r \in \Delta_{D}$ on which $t^{\mathcal{I}}(u, r)$ is defined.

Given arbitrary t-norm $\otimes$, t-conorm $\oplus$, negation function $\ominus$ and implication function $\Rightarrow$, the fuzzy interpretation function is extended to complex concepts and fuzzy axioms as below:

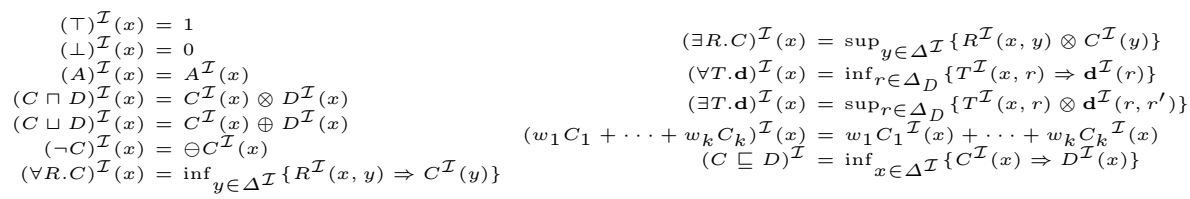

A fuzzy interpretation $\mathcal{I}$ satisfies (is a model of) a fuzzy statement $\langle\alpha, n\rangle$ iff $\alpha^{\mathcal{I}} \geq n$. The notions of logical consequence, best entailment degree and best satisfiability degree of $\alpha$ are as for Section 2. We additionally define the Best Satisfiability Degree (BSD) [3] of a concept $C$ w.r.t. a fuzzy KB $\mathcal{K}$ as

$$
\operatorname{bsd}(\mathcal{K}, C)=\sup _{\mathcal{I} \models \mathcal{K}} \sup _{x \in \Delta^{\mathcal{I}}} C^{\mathcal{I}}(x) \text {. }
$$

MCDM and Fuzzy DLs. We next provide some examples, illustrating how to encode some simple (fuzzy) MCDM problems in fuzzy DLs, showing the potential of our approach.

Example 1. The MCDM problem consists of four alternatives and five criteria for an electrical power dispatching system in the case of shortage of electrical power2 2 . The four alternatives correspond to four regions of a city to which to give priority. The five criteria correspond to $C_{1}$ (Residential area), $C_{2}$ (Shopping centers), $C_{3}$ (Clubs and recreation centers), $C_{4}$ (Educational centers), and $C_{5}$ (Medical urgent care centers). We normalize the performance values according to $q_{i j}=w_{j} \cdot\left(a_{i j} / \sum_{l=1}^{n} a_{l j}\right)$. The decision matrix together with the matrix of the $q_{i j}$ 's is shown below:

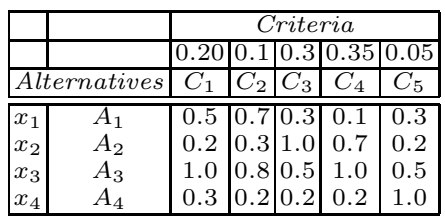

$q_{i j}=\left(\begin{array}{lllll}0.0455 & 0.0333 & 0.0391 & 0.0149 & 0.0073 \\ 0.0182 & 0.0143 & 0.1304 & 0.1043 & 0.0049 \\ 0.0909 & 0.0381 & 0.0652 & 0.1489 & 0.0122 \\ 0.0273 & 0.0095 & 0.0261 & 0.0298 & 0.0244\end{array}\right)$

${ }^{2}$ The use case is inspired by http://med.ee.nd.edu/MED11/pdf/papers/t3-013.pdf 
Now, we further assume that each of the five criteria has dynamic, time dependent electricity demand on the day time (18-24) as depicted below (values are normalized to one).

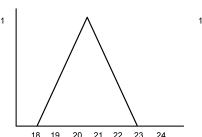

$C_{1}$

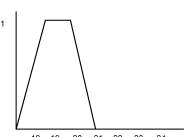

$\mathrm{C}_{2}$

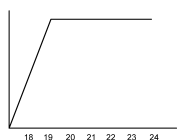

$C_{3}$

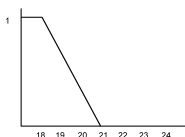

$\mathrm{C}_{4}$

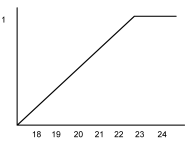

$C_{5}$

We model this situation by assuming that we have in the KB the axioms:

$C_{1}=$ ResidentialArea $\sqcap \exists$ hasDemand.tri $(18,20.50,23) \quad C_{2}=$ ShoppingCenter $\sqcap \exists$ has Demand.trap $(17,19,20,21)$ $\begin{array}{ll}C_{3}=\text { ClubRicreationCenter } \sqcap \exists \text { hasDemand.rs }(17,19) & C_{2}=\text { ShoppingCenter } \sqcap \exists \text { hasDemand.trap }(17,19,20, \\ C_{5}=\text { MedicalUrgentCareCenter } \sqcap \exists \text { hasDemand.rs }(23,24) & C_{4}=\text { EducationalCenter } \sqcap \exists \text { hasDemand.Is }(19,21)\end{array}$

where hasDemand is a functional concrete feature. We now define the four alternatives $A_{i}$ as the following weighted concepts:

$$
A_{i}=q_{i 1} \cdot C_{1}+q_{i 2} \cdot C_{2}+q_{i 3} \cdot C_{3}+q_{i 4} \cdot C_{4}, \text { for } i=1,2,3,4 .
$$

The final rank value of alternative $A_{i}$ w.r.t. a knowledge base $\mathcal{K}$, denoted $r v\left(\mathcal{K}, A_{i}\right)$ is defined as $r v\left(\mathcal{K}, A_{i}\right)=b s d\left(\mathcal{K}, A_{i}\right)$, i.e. we compute its maximal satisfiability degree. It is thus, easily verified that this extents the WSM to the fuzzy DL case. Finally, the optimal alternative is $A^{*}=\arg \max _{A_{i}} r v\left(\mathcal{K}, A_{i}\right)$. Now, assume that we have a shortage of power between time 19-20. It can be verified that (the values have been computed using the fuzzy DL reasoner FUZZYDL 3]) $r v\left(\mathcal{K}, A_{1}\right)=$ $0.11625, r v\left(\mathcal{K}, A_{2}\right)=0.25628, r v\left(\mathcal{K}, A_{3}\right)=0.28856, r v\left(\mathcal{K}, A_{4}\right)=0.07632$, and, thus, the ranking of the alternatives is $A_{3} \succ A_{2} \succ A_{1} \succ A_{4}$ and the optimal alternative is $A^{*}=A_{3}$.

Example 2. The following example is a simplified version of 4 and is about landfill siting. We have to select among two sites, Site $_{1}$, Site $_{2}$, according to two criteria ( $T I$-Transportation Issues, and $P N$-Public Nuisance) and there are two experts $\left(E_{1}, E_{2}\right)$. The decision matrix of the experts is shown below:

\begin{tabular}{|c|c|c|c|c|c|c|c|}
\hline$\overline{E_{1}}$ & & \multicolumn{2}{|c|}{ Criteria } & \multirow[t]{2}{*}{$E_{2}$} & & \multicolumn{2}{|c|}{ Criteria } \\
\hline & & 0.48 & 0.52 & & & 0.52 & 0.48 \\
\hline \multicolumn{2}{|c|}{ Alternatives } & $C_{1}$ & $C_{2}$ & \multicolumn{2}{|c|}{ Alternatives } & $C_{1}$ & $C_{2}$ \\
\hline$x_{1}$ & $\overline{A_{1}}$ & $\overline{\operatorname{tri}(0.6,0.7,0.8)}$ & $\operatorname{tri}(0.9,0.95,1.0)$ & $x_{1}$ & $\overline{A_{1}}$ & $\operatorname{tri}(0.55,0.6,0.7)$ & $\operatorname{tri}(0.4,0.45,0.5)$ \\
\hline$x_{2}$ & $A_{2}$ & $\operatorname{tri}(0.6,0.7,0.8)$ & $\operatorname{tri}(0.4,0.5,0.6)$ & $x_{2}$ & $A_{2}$ & $\operatorname{tri}(0.35,0.4,0.45)$ & $\operatorname{tri}(0.5,0.55,0.6)$ \\
\hline
\end{tabular}

Note that this time, the performance of the alternatives is defined in terms fuzzy numbers. We may model the scenario as follows. For each expert $k=1,2$, for each alternative $i=1,2$ and for each criteria $j=1,2$, we define the concept

$$
P_{i j}^{k}=\exists \text { hasScore. } a_{i j}^{k},
$$

where hasScore is a concrete feature and $a_{i j}^{k}$ is, according to expert $k$, the performance of alternative $i$ with respect to criteria $j\left(a_{i j}^{k}\right.$ is the fuzzy number in the matrix). Now, for each expert $k$ and alternative $i$, we define the weighted concept

$$
A_{i}^{k}=w_{1}^{k} \cdot P_{i 1}^{k}+w_{2}^{k} \cdot P_{i 2}^{k}
$$

which takes into account also the weight $w_{j}^{k}$ of expert $k$ for criteria $j$. Finally, we combine the two experts outcome, by defining the weighted concept 


$$
A_{i}=0.5 \cdot A_{i}^{1}+0.5 \cdot A_{i}^{2} .
$$

Note that we rate both experts equally (this may be changed, of course). The final rank value of alternative $A_{i}$ w.r.t. a knowledge base $\mathcal{K}$ and the optimal alternative is determined as for Example 1. It can be verified (using again the fuzzy DL reasoner FUZZYDL [3]) that $r v\left(\mathcal{K}, A_{1}\right)=0.26$ and $r v\left(\mathcal{K}, A_{2}\right)=0.37$ and, thus, the ranking of the alternatives is $A_{2} \succ A_{1}$ and the optimal alternative is $A^{*}=A_{2}$.

\section{Conclusions}

We have made an initial step in addressing MCDM within fuzzy DLs and, thus, towards a fuzzy knowledge-assisted approach to decision making. Our aim here was exploratory on the argument and a more in depth investigations need to be addressed, of course.

There are several points that may be of interest for future research: $(i)$ each alternative is indeed a fuzzy set and, so far, we order alternatives according to the best satisfiability degree. Other methods can be explored by relying on the fuzzy membership function of the alternatives, e.g. using defuzzification methods; ( $i i$ ) fully exploit fuzzy numbers as performance and weight values in decision matrixes; (iii) for illustrative purposes, we have just considered a simple, though widely used, basic MCDM method, namely the weighted sum method. The MCDM literature (inclusive their fuzzy MCDM variants) is quite large, so it will be of interest whether and how other methods can be integrated within fuzzy DLs as well. E.g., for illustrative purposes, we considered the weighted sum method, though in general other fuzzy aggregation operators may be needed to combine the multiple performance values into an aggregated value (e.g., to cope with criteria interdependence/conflict); (iv) exploit the fact that we may express background/domain knowledge within fuzzy DLs (e.g., in Example1 we may include an Urban Ontology to formalize the problem, which is part of larger GIS system; a similar argument applies to Example 2 as well); $(v)$ fuzzy DLs are parametric with respect to t-norm, t-conorm, etc. Choosing, e.g., appropriate fuzzy connectors, as well as appropriate fuzzy aggregation operators, is clearly application specific and may bring to different results and, thus, these issue needs both theoretical and empirical investigations in our setting as well.

\section{References}

1. Baader, F., Calvanese, D., McGuinness, D., Nardi, D., Patel-Schneider, P.F. (eds.): The Description Logic Handbook: Theory, Implementation, and Applications. Cambridge University Press, Cambridge (2003)

2. Bobillo, F., Delgado, M., Gómez-Romero, J.: Delorean: A reasoner for fuzzy OWL 1.1. In: Proceedings of the 4th International Workshop on Uncertainty Reasoning for the Semantic Web (URSW 2008). CEUR Workshop Proceedings, vol. 423, p. 10 (2008) 
3. Bobillo, F., Straccia, U.: fuzzyDL: An expressive fuzzy description logic reasoner. In: International Conference on Fuzzy Systems (FUZZ 2008), pp. 923-930. IEEE Computer Society, Los Alamitos (2008)

4. Chang, N.B., Parvathinathan, G., Breeden, J.B.: Combining GIS with fuzzy multicriteria decision-making for landfill siting in a fast-growing urban region. Journal of Environmental Management 87(1), 139-153 (2008)

5. Hájek, P.: Metamathematics of Fuzzy Logic. Kluwer, Dordrecht (1998)

6. Kahraman, C.: Fuzzy Multi-Criteria Decision Making: Theory and Applications with Recent Developments. Springer, Dordrecht (2008)

7. Klir, G.J., Yuan, B.: Fuzzy sets and fuzzy logic: theory and applications. PrenticeHall, Inc., Upper Saddle River (1995)

8. Lukasiewicz, T., Straccia, U.: Managing uncertainty and vagueness in description logics for the semantic web. Journal of Web Semantics 6, 291-308 (2008)

9. Stoilos, G., Simou, N., Stamou, G., Kollias, S.: Uncertainty and the semantic web. IEEE Intelligent Systems 21(5), 84-87 (2006)

10. Straccia, U.: Reasoning within fuzzy description logics. Journal of Artificial Intelligence Research 14, 137-166 (2001)

11. Straccia, U.: Description logics with fuzzy concrete domains. In: Bachus, F., Jaakkola, T. (eds.) 21st Conference on Uncertainty in Artificial Intelligence (UAI 2005), Edinburgh, Scotland, pp. 559-567. AUAI Press (2005)

12. Straccia, U.: A fuzzy description logic for the semantic web. In: Sanchez, E. (ed.) Fuzzy Logic and the Semantic Web. Capturing Intelligence, ch. 4, pp. 73-90. Elsevier, Amsterdam (2006)

13. Triantaphyllou, E.: Multi-Criteria Decision Making Methods: A Comparative Study. Kluwer Academic Publishers, Dordrecht (2000) 